\title{
VOCES INSCRITAS EN LOS CUERPOS FEMENINOS ${ }^{1}$
}

\begin{abstract}
Alexandra $\mathrm{M}^{\mathrm{a}}$ Ríos Marín ${ }^{2}$
Voces inscritas en los cuerpos femeninos

Resumen: El concepto de salud ha ido evolucionando desde el concepto rígido y reduccionista que limita la salud a una visión simple y biologicista, hacia un concepto más amplio que integra la dimensión biológica (física), psicológica y social, en cuanto afectan la salud no sólo de un individuo sino de todo un colectivo. Una de las principales críticas del feminismo al paradigma biomédico es el androcentrismo que se extiende a todos los niveles propios del ámbito médico-científico, así como la alta tendencia hacia la medicalización de las mujeres o la banalización de sus síntomas e invisibilización de sus problemas en salud. El objetivo de este artículo es realizar un análisis desde la antropología feminista y el modelo de desigualdades sociales en salud acerca de las diferentes formas de violencias que se inscriben en los cuerpos de las mujeres y la respuesta del discurso biomédico ante sus demandas. El análisis se ha realizado a través de las voces de diferentes sobrevivientes de violencia de género en los siguientes contextos estudiados: prostitución y/o trata de personas con fines de explotación sexual y violencia sexual en el conflicto armando; testimonios obtenidos a través de la escucha activa y la observación participante, en etnografías realizadas tanto en la provincia de Almeria como en Colombia.
\end{abstract}

Palabras clave: Desigualdades sociales, Género, Prostitución, Salud, Trata de personas, Violencias de género.

\section{Voices inscribed in female bodies}

Abstract: The concept of health has evolved from the rigid and reductionist concept that limits health to a simple and biologicist vision, towards a broader concept that integrates the biological (physical), psychological and social dimension, as they affect the health not only of an individual but of a whole collective. One of the main criticisms of feminism to the biomedical paradigm is the androcentrism that extends to all levels of the medical-scientific field, as well as the high tendency towards the medicalization of women or the banalization of their symptoms and the invisibility of their problems in Health. The objective of this article is to carry out an analysis from the feminist anthropology and the model of social inequalities in health about the different forms of violence that are inscribed in the bodies of women and the response of the biomedical discourse to their demands. The analysis carried out with the voices of different survivors of gender violence in the following contexts studied: prostitution and / or trafficking of persons for the purpose of sexual exploitation and sexual violence in the armed conflict; testimonies obtained through active listening and participant observation, in ethnographies carried out both in the province of Almeria and in Colombia.

Key words: Social inequalities, Gender, Prostitution, Health, Human Trafficking, Gender Violence.

\footnotetext{
${ }^{1}$ Fecha de recepción: 10/10/2019.

Fecha de aceptación: 29/11/2019.

2 Profesora Sustituta Interina, Departamento de Psicología, Universidad de Almería, España; $\square$ alexrios@ual.es.
} 


\section{Introducción}

La violencia por motivos de género ha sido definida por el Comité para la Eliminación de la Discriminación Contra la Mujer (CEDAW) como la violencia que es dirigida hacia una persona por razón de su género o sexo, que incluye actos que causan daño o sufrimiento físico, mental o sexual; la amenaza de tales actos, la coerción y otras formas de privación de la libertad (CEDAW 1979). Además, este tipo de violencia constituye una violación de los derechos humanos y perpetúa los estereotipos y los roles de género que niegan la dignidad humana de las personas y limita su desarrollo individual, personal y comunitario (ACNUR 2012).

Una de las formas más lesivas de violencia de género es la violencia sexual, definida como: "la violación, la esclavitud sexual, la prostitución forzada, los embarazos forzados, la esterilización forzada o cualquier otra forma de violencia sexual de gravedad comparable que se cometa contra las mujeres, los hombres o los niños" (ONU 2012). La violencia sexual deja un efecto devastador en los cuerpos de las mujeres víctimas, que se expresa en su salud. Los cuerpos hablan lo que sus labios callan. La violencia sexual también hace mella en sus relaciones personales, familiares y comunitarias (MPDL 2017), generando un estrés permanente al que tienen que hacer frente las mujeres, en la mayoría de las ocasiones, en soledad y carentes de redes de apoyo social; así mismo se dificulta la reparación de los impactos en su salud bio-psico-social, debido a las desigualdades sociales que enfrentan según su contexto y a la violencia institucional que no las considera interlocutoras válidas de sus dolencias, exigiendo pruebas médicoforenses para constatar las lesiones de la violencia sexual en sus cuerpos. 
La incorporación de la perspectiva de género en las ciencias sociales, gracias a las sucesivas olas de teorías feministas, han permitido la investigación y la visibilización de la importancia de articular al discurso de los derechos humanos la equidad de género, analizando el continuum de la violencia en el que se ven inmersas las mujeres y niñas. La violencia de carácter sexual se ve exacerbada por los conflictos armados (Salcedo, 2013, MPDL 2017) en los que es frecuente la proliferación de una industria del sexo a escala global que favorece el tráfico y la trata de personas con fines de explotación sexual: según los últimos datos presentados por Naciones Unidas el $70 \%$ de las víctimas de la trata son mujeres y niñas (ONU 2019).

La antropología feminista, así como las teorías de los determinantes sociales generadores de las desigualdades en salud (Marmot, Friel, Bell, Houweling, y Taylor 2008; Marmot, Allen, Bell, Bloomer, y Goldblatt 2012), han constituido el marco teórico para las investigaciones que han dado origen a este artículo, analizando dos estudios etnográficos independientes sobre un denominador común: la atención en salud de las mujeres víctimas/sobrevivientes de la violencia sexual. El primer estudio: Migraciones, Género y Salud: un estudio antropológico de las salud e integración social de las mujeres migrantes extranjeras que ejercen la prostitución en la provincia de Almería (Ríos 2015). Y el segundo estudio: Cicatrices de la guerra en las colombianas. Los impactos de la violencia sexual en Montes de María, realizado por la ONGD Española Movimiento por la Paz el Desarmen y la Libertad (MPDL 2017). 


\section{Aportes de la antropología feminista al modelo biomédico}

La antropología feminista ha cuestionado el paradigma biomédico a través del proceso de desconstrucción de los enfoques esencialista y naturalista, develando la invisibilidad de las mujeres a través de este discurso y sus contradicciones androcéntricas. Las teorías feministas, como instrumentos de producción de conocimiento (Haraway 1988), han posibilitado el cuestionamiento no sólo de las diferencias naturalizadas y rígidamente establecidas entre mujeres y varones, sino también de las propias categorías y conceptos teóricos en los que se sustenta la ciencia moderna, aportando así otra mirada a la construcción del conocimiento, en la cual se tiene en cuenta el sistema de género y se visibiliza la diferencia sexual y la condición jerarquizada de las mujeres a partir de las condiciones sociales, económicas y políticas que reproducen y mantienen las relaciones de poder:

El análisis de género significa tener en cuenta la falta de poder de las mujeres sobre su salud, motivado por su posición de subordinación histórica en la sociedad, lo que ha supuesto y conlleva su relegación al ámbito doméstico y su exclusión de la vida pública y del poder político. Esto supone que su capacidad de tomar decisiones sobre su propia salud, la de su familia y la de la comunidad ha estado limitada y es prácticamente inexistente en algunos países del mundo. (Mazarrasa 2001: 84)

De este modo, en el discurso biomédico, las mujeres tampoco han sido “interlocutoras válidas" (Juliano 2004) sobre lo que ocurre con sus cuerpos. La hegemonía del discurso biomédico ha dado cuenta de los procesos de salud y enfermedad de las mujeres a través de la biología de los cuerpos masculinos, sin tener en cuenta las diferencias. Así, el paradigma biomédico explica la enfermedad como un trastorno 
anatómico y morfo-fisiológico ligado fundamentalmente a la biología. La enfermedad es una experiencia clínica definida por el médico, que no incluye el contexto sociocultural como elemento importante para aclarar la causalidad de las enfermedades. Se presupone que los principales factores causantes de la enfermedad son orgánicos y entre ellos tiene un peso fundamental la genética y la constitución del individuo.

Desde esta perspectiva biomédica, el saber está centrado en el/la facultativo-a, que se constituye en sujeto observador-a del paciente y, a través de sus conocimientos clínicos y la lectura de signos y síntomas, descifra el significado de la lesión orgánica, teniendo en cuenta la desviación estadística de la normalidad. Además, en situaciones extremas como en los contextos de agresión sexual donde acontece la violencia son las-os especialistas que se hacen cargo, a través de las pruebas periciales quienes determinan la continuidad o no de la atención y reparación integral de las mujeres víctimas:

Entonces me dijeron que tenía que ir a Medicina Legal con un documento que ellos me dieron y cuando fui a Medicina Legal, claro como ya habían pasado varios días, no me tomaron muestra; el doctor me dijo: "no te puedo tomar muestra", lo único es que puedes ir a algo de psicología, pero que ellos me llamarían, al sol de hoy después de 5 años nunca me llamaron (Luisa, 33 años, Colombia). (MPDL, 2017)

En el informe final de la investigación realizada por MPDL en Colombia, han denunciado cómo las pruebas médico-forenses están por encima de la Ley colombiana que debe garantizar la reparación integral de las víctimas.

Centrar toda la prueba de la violencia sexual en los impactos físicos, principalmente en el aparato reproductor femenino, es una visión anuladora de la mujer, ya que las mujeres no son solo "vagina"; la violencia sexual deja múltiples impactos, tal como hemos detallado en los resultados anteriores; la mujer es un ser humano integral "biopsico-social" y trascendente -entiéndase creyente, valorativo, espiritual-. No se entiende, entonces, que la Ley 1448 de 2011 reconozca la importancia del enfoque psicosocial y diferencial en la atención a las víctimas de violencia en el marco del conflicto armado, y que dichos enfoques no sean implementados en el momento del acceso de las víctimas a sus derechos, a la asistencia y a la reparación integral. (MPDL 2017: 147) 
Una de las principales críticas del feminismo a este paradigma biomédico es el androcentrismo que se extiende a todos los niveles propios del ámbito médico-científico, así como la alta tendencia hacia la medicalización de las mujeres, lo "que supone un control social e ideológico sobre la población, a través fundamentalmente de la estigmatización y regulación de ciertos comportamientos y la negatividad asociada a determinados padecimientos; y a la misma definición de lo que es enfermedad y de lo que no lo es, de quienes son asistibles y en qué condiciones" (Esteban 2004: 35). Según Esteban (2004), el sector salud es el ejemplo por excelencia de la tendencia a separar radicalmente a las mujeres y a los varones, y a dar explicaciones acerca de la salud de unas en función de otros, en base a su diferencia biológica y sexual, sin tener en cuenta el aspecto relacional, social, cultural y otras variables que inciden en las desigualdades entre ambos géneros y su repercusión en la salud, como por ejemplo, la condición de extranjería de las mujeres en contextos en prostitución y/o víctimas de trata.

Ortiz (1993), por su parte, expresa cómo el discurso biomédico se ha constituido por mucho tiempo en un poder experto y productivo, que se ha naturalizado a través de una percepción absolutamente esencialista y biologicista del cuerpo humano, sobre todo el cuerpo de la mujer:

En realidad, voy a hablar de discursos médicos sobre la mujer, siendo como es la mujer en medicina una categoría biológica, a veces patológica, única, poco susceptible de variedad. El discurso, por una parte, es también único y de carácter masculino y patriarcal. Es masculino porque hasta fechas muy recientes lo han construido exclusivamente varones desde una óptica que toma lo masculino como la norma y lo femenino como lo otro, y es patriarcal porque sirve a los intereses de un sistema de dominación masculino. Pero si digo discursos, en plural, es porque quiero subrayar que, dentro de este marco genérico, la diversidad también existe y el discurso ha adoptado y adopta diversas formas dependiendo del momento histórico y del contexto social, político y biográfico de quienes lo han construido (Ortiz 1993: 109). 
La biopolítica (Foucault 1994) pone de manifiesto que el poder no sólo lo ejerce el monarca o el gobierno sino también los discursos expertos que las diversas ciencias vienen desarrollando desde la modernidad, puesto que se trata de un poder que es experto y productivo a la vez, ya que actúa a través del establecimiento de las normas de comportamiento. A tenor de esta realidad, la organización Women's Link presentó una querella en el 2017 por un delito de odio contra el psicólogo forense que emite la mayoría de los informes periciales para la Administración de Justicia en Asturias:

En los informes que elabora en juicios por violencia de género y por abusos sexuales "desacredita el testimonio de las mujeres y de sus hijos e hijas basándose en prejuicios y estereotipos de género, como que las mujeres manipulan a sus hijos e hijas para dañar a sus parejas o exparejas". Es decir, "no se las cree por el hecho de ser mujeres y se las trata como unas mentirosas que se aprovechan de los procedimientos judiciales". (Women's Link 2018)

En el mismo sentido, Esteban (2001) resalta cómo la crítica feminista en materia de salud ha tenido como eje de trabajo prioritario el ámbito médico-científico. Aunque, en general, hay una tendencia a considerar estos aspectos como sesgos, "no se trata de simples desviaciones o imperfecciones del sistema médico, sino que es una cuestión estructural al propio sistema, que hay que recontextualizar y repensar en su totalidad" (Esteban 2001:35). La doctora Carmen Valls (2006, 2011), por su parte, confirma cómo "esta cuestión estructural ha hecho que las mujeres sean invisibles para la atención sanitaria en el diagnóstico y el tratamiento de muchas enfermedades, que sus síntomas sean confundidos, minimizados o no bien diagnosticados, y que sus quejas sean frecuentemente atribuidas a etiología psicológica o psicosomática” (Valls 2011: 22).

Valls enfatiza la trascendencia de los estereotipos de género que han sesgado las ciencias de la salud, dejando a las mujeres invisibles respecto a su realidad bio- psico- 
social, "hacen pasar por ciencia las mismas operaciones que se han practicado sobre el cuerpo de las mujeres, las tres reglas del patriarcado: a) La naturalización de la diferencia sexual como inferioridad; b) la fragmentación del cuerpo y de la experiencia y c) la objetualización de los sujetos" (Valls 2011:22).

Las mujeres en contextos de prostitución y/o victimas de trata para la explotación sexual son numerosas como víctimas para las estadísticas oficiales, pero escasas como titulares de derechos e invisibles en sus problemáticas de salud bio-psico-social; las tres reglas del patriarcado se inscriben en los cuerpos de las mujeres, como lo resalta Juliano (2010):

Las mujeres extranjeras se encuentran desprotegidas dentro el marco de las políticas públicas que deberían encargarse de que sus condiciones de vida mejoren, de que tengan mejores posibilidades laborales, mayores canales de regularización de su situación administrativa, principal causa por la cual las mujeres extranjeras encuentran en la industria del sexo una estrategia de supervivencia. No solo no se encuentran representadas, ni son escuchadas en los diferentes estamentos públicos sino que su estrategia de supervivencia es rechazada y constituye factor de discriminación, no solo social sino que, como he denunciado anteriormente, la violencia institucional no reconoce sus necesidades reales y limita el acceso a los recursos existentes. (Juliano 2010)

En España la organización Médicos del Mundo atendió en 2018 a 8.993 personas en situación de prostitución, de las cuales 8.714 eran mujeres, 90\% de ellas, mujeres extranjeras (MDM 2018). Dedicarse a una actividad fuertemente estigmatizada socialmente incrementa en las personas que ejercen la prostitución y/o que son víctimas de la trata para la explotación sexual el estrés psicológico en sus vidas, ligado a los procesos y duelos migratorios que se desarrollan en contextos cuya atmósfera es la violencia de género (Achostegui 2004; Sayed-Ahmad 2008; Bungay, Halpin, Halpin, Jonhston y Patrick 2012; Ríos, 2015). 
Las políticas sanitarias y sociales que van dirigidas a las mujeres en contextos de prostitución y/o víctimas de trata, deberán reconocer la importancia de las desigualdades sociales en salud que enfrentan estas personas para diseñar intervenciones más acordes a las situaciones psicosociales complejas que hacen mella en la salud bio-psico-social de las mujeres, y dificultan los adecuados procesos de inclusión social (Ríos 2015).

\section{Los sesgos de género y la desigualdad en salud}

El planteamiento erróneo de igualdad o de diferencia entre mujeres y varones, en cuanto a su naturaleza, comportamiento e ideas acerca de su salud, puede generar, según Ruiz (2001), una conducta desigual en la atención en salud. La investigación y los servicios de salud pueden incurrir en sesgos discriminatorios de género, a través de dos enfoques:

En primer lugar, asumir que las situaciones de salud de las mujeres y los varones y sus riesgos son similares, cuando de hecho no lo son, y, en segundo lugar, establecer las diferencias cuando realmente son similares. Las acciones y la investigación que siguen a ambas aproximaciones pueden influir en los resultados de la salud de las mujeres de forma negativa. (Ruiz 2001:164)

Respecto a la primera cuestión señalada por Ruiz (2001), un hecho que ha reforzado la idea de que no existen diferencias de género en salud es la tendencia a la realización de ensayos clínicos contando exclusivamente con los varones y asumiendo con posterioridad que los resultados son generalizables a las mujeres (Ruiz 2001; Valls 2011). Dicha práctica ha dañado la salud de las mujeres en lugar de protegerla. De la misma manera se ha procedido en la investigación de enfermedades comunes y en el análisis de factores de 
riesgo y protección de la salud, analizando los factores relacionados con los varones. La importancia de la cultura, el contexto político y social, la clase social, la edad, el sistema de valores de las personas, el significado de enfermedad o de salud, y las relaciones de género que se establecen en el proceso de salud-enfermedad, constituyen factores fundamentales a tener en cuenta en toda investigación científica relacionada con la salud de las mujeres y de los varones, más aún cuando estamos abordando realidades tan complejas como las consecuencias para la salud de la violencia sexual.

En lo que se refiere al segundo enfoque, la diferencia se centra especialmente en la función reproductora de las mujeres. "Las relaciones de la medicina con la salud reproductiva explicitan los estereotipos de género, no en este caso por la invisibilidad, sino por visibilizarlos siempre como un problema, como una enfermedad que hay que tratar" (Valls 2011: 326). Así mismo, se explicitan las ITS y VIH/SIDA en la mujeres víctimas de violencia sexual, obviando otras problemáticas en su salud bio-psico-social que para ellas son más importantes; más aún, cuando la literatura internacional y nacional al respecto han constatado la baja incidencia de ITS y VIH/SIDA entre las mujeres que ejercen la prostitución (Cabrerizo, Barroso y Rodríguez 2013; Ríos 2015).

La expansión de los programas de prevención y de reducción de riesgos en España es otro de los factores que han contribuido a ésta baja incidencia; sin embargo los programas de prevención de ITS y VIH/SIDA, se centran exclusivamente en las mujeres prostituidas, y no tanto interés y acento en el prostituidor, en los clientes de la prostitución (Wong et al., 2012; Gaines et al., 2013; Ríos 2015). Así lo detalla Ríos en su investigación dentro de la provincia de Almería:

A pesar de esta importante presencia de varones inmigrantes y autóctonos en los escenarios de prostitución, durante el desarrollo del trabajo de campo, no se conoció ningún programa de prevención de las ITS/VIH, destinado a los clientes de la 
industria sexual, lo que evidencia que las conductas de riesgo de ITS/VIH y protección inadecuada, han sido moldeadas por factores de género y poder, que se establecen en la relación asimétrica entre mujeres que ejercen la prostitución, parejas afectivas y clientes. (Ríos 2015:349)

Este hincapié por visibilizar solo la salud sexual y reproductiva de las mujeres en prostitución, utilizándolas como "Cuidadoras" y "Responsables" de los cuerpos de sus clientes (Ríos 2015) no posibilita realizar una intervención en salud más integral que reconozca otras problemáticas:

Las mujeres extranjeras que ejercen la prostitución en exteriores están expuestas a contaminantes en sus lugares de residencia y de trabajo, principalmente las de origen africano, subsaharianas y magrebíes que residen entre invernaderos, consumen agua no potable de las balsas y de los riegos de los sectores agrícolas. La exposición bajo plásticos, al intenso calor en el verano, y al frío en el invierno, deja huella en sus pieles. Las lesiones físicas de la piel son frecuentes también, en las personas que ejercen en exteriores como calles, carreteras comarcales y carreteras entre invernaderos expuestas, sin protección, a largas horas de luz solar y a diferentes inclemencias climáticas, según la estación del año. (Ríos 2015: 347)

Estos enfoques sesgados por el género también se presentan en la atención de mujeres y varones en relación con su bienestar psicológico. La dimensión psíquica estaba invisibilizada en las mujeres y sólo se habían trabajado con los síntomas físicos, a los que se les intentaba encontrar correlación con alguna enfermedad orgánica (Valls 2011). La incorporación de la perspectiva de género dependerá del paradigma rector del profesional en relación a la salud mental. Según Távora (2001), existen tres perspectivas desde donde abordar la salud mental de las personas: una perspectiva psicopatológica, una perspectiva psicosocial y una perspectiva de género. Así nos encontramos con un primer grupo de profesionales que abordan los problemas de los-as pacientes desde una perspectiva exclusivamente psicopatológica. Esta perspectiva es muy común cuando se aborda la violencia sexual ligada al ejercicio de la prostitución, casos en los que a las mujeres les 
suelen aplicar determinados estereotipos del estilo: "Si es prostituta seguramente la violaron de pequeña..."; desconocen la realidad de la trata de mujeres con fines de explotación sexual y los impactos para la salud mental de las víctimas.

En un segundo grupo, encontramos a los profesionales que tienen una perspectiva más relacional, más psicosocial del sufrimiento psicológico de las mujeres. Estos profesionales de la salud mental establecen relaciones entre la manera de vivir y el sufrimiento, hacen ofertas grupales para apoyar a las mujeres y les ofrecen terapias de apoyo (Távora 2001: 201). Sin embargo, integrar la perspectiva de género en salud mental implica, por parte de los-as profesionales, un análisis de la construcción social de lo que significa ser mujer o ser varón, y de cómo esa construcción facilita o no la aparición de ciertas psicopatologías. No se trata sólo de analizar la relación entre los estilos de vida y el sufrimiento, sino también de profundizar en por qué existe una organización social que, mientras a unos les permite desarrollarse como sujetos -en este caso a los varones-, a otras les facilita la anulación de la propia individualidad (Puleo 2002). Las situaciones de desigualdad, la edad, la clase social, el ejercicio de la prostitución como factor de riesgo, la experiencia de un proceso migratorio, las relaciones de dependencia con los varones, la relación entre inferioridad y pérdida de la autoestima, el papel de la maternidad, son temas centrales para las personas profesionales en salud mental que integran la perspectiva de género (Távora 2001).

\section{Conclusiones}

Es fundamental implementar el enfoque de género en salud que permita promover iniciativas encaminadas a corregir las inequidades en el acceso a la atención sanitaria y 
en las respuestas del sistema de salud a las mujeres y niñas víctimas de la violencia sexual en diferentes contextos.

Los sesgos de género en el sistema de salud no son elementos aislados sino que son parte de una cultura androcéntrica, por lo tanto, es necesario que todo el personal sanitario responsable de la atención y cuidados de la salud bio-psico-social de las mujeres víctimas de violencia sexual integren a su saber específico una lectura que incluya el contexto social y articule las teorías explicativas de las desigualdades de género y el análisis de las desigualdades sociales en salud, con el objetivo de contribuir a la reparación integral de las victimas/sobrevivientes de la violencia sexual.

\section{Referencias bibliográficas}

ACNUR. 2012. Identificación, Prevención, y Respuesta frente a la violencia sexual basada en género, en el marco del conflicto armado, en el distrito de Buenaventura. Documento Final. Diagnóstico Participativo. Buenaventura. Colombia: ACNURFUNDACION AKINA ZAJI SAUDA.

Bungay, V., Halpin, M., Halpin, P., Jonhston, C., Patrick, D. (2012). "Violence in the massage parlor industry: experiences of Canadian-born and immigrant women". Health Care Women Int, 33(3):262-84.

Cabrerizo, María Jesús, Barroso, María Pilar y Rodríguez Pelayo Rafael. 2013. "Infecciones de transmisión sexual en mujeres que ejercen la prostitución en Almería". Actualidad médica, 98(789):74-77.

Comité del CEDAW. 1979. Convención para la eliminación de todas las formas de discriminación contra la mujer. Resolución de la Asamblea General A/RES/34/180, 18 diciembre 1979.

Esteban, Mari luz. 2004. Antropología del cuerpo. Barcelona: Bellaterra.

Foucault, Michel. 1994. Naissance de la biopolitique. Cours au Collège de France. 19781979. París: Gallimard-Seuil. 
Gaines, T., Rudolph, A., Brouwer, K., Strathdee, S., Lozada, R., Martinez, G y Rush, M. 2013. "The longitudinal association of venue stability with consistent condom use among female sex workers in two Mexico-USA border cities". Int J STD AIDS, 24(7):523-9.

Haraway, Donna (Autumn 1988). Situated Knowledges: The Science Question in Feminism and the Privilege of Partial Perspective. Feminist Studies. 14 (3): 575599.

Juliano, Dolores. 2004. Excluidas y marginales: Cátedra.

2010. Ponencia en Universidad de Málaga (Transcripción de entrevista).

Marmot, M; Allen, J; Bell, R; Bloomer, E y Goldblatt, P. WHO European review of social determinants of health and the health divide. Lancet. 2012; 380: 1011-29.

Marmot, M; Friel, S; Bell, R; Houweling, TA; y Taylor, S; Commission on Social Determinants of Health. Closing the gap in a generation: health equity through action on the social determinants of health. Lancet. 2008; 372:1661-9

Mazarrasa, Lucía. 2001. "Estrategias de promoción de la salud”. En Miqueo, Consuelo., Tomás, Concepción; Tejero, Cruz., Barral, María José., Fernández, Teresa y Yago, Teresa (eds.) Perspectivas de género en salud. Fundamentos científicos y socioprofesionales de diferencias sexuales no previstas. Madrid: Minerva.

Médicos del Mundo (MDM). 2018 Memorias de actividades Médicos del Mundo 2018. https://www.medicosdelmundo.org/actualidad-ypublicaciones/publicaciones/ memoria-de-actividades-de-medicos-del-mundo-2018 [08 octubre 2019].

Movimiento por la paz el desarme y la libertad (MPDL). 2017. Cicatrices de la guerra en las colombianas. Los impactos de la violencia sexual en Montes de María. MPDL

Organización de Naciones Unidas (ONU). 2019. La trata de personas aumenta en zonas de conflicto. https://news.un.org/es/story/2019/01/1449042 [14 abril 2019].

ONU MUJERES. 2012. Análisis de Género y Conflicto. Entidad de las Naciones Unidas para la igualdad de género y el empoderamiento de las Mujeres. NY.

Ortiz, T. 1993. "El discurso médico sobre las mujeres en la España del primer tercio del siglo veinte". En López, M.T. Las mujeres en Andalucía. Actas del II Encuentro Interdisciplinar de Estudios de la Mujer en Andalucía. Málaga: Diputación Provincial de Málaga.

Puleo, Alicia. 2002. Feminismo y ecología. "Un repaso a las diversas corrientes del ecofeminismo". El Ecologista, 31:36-39.

Ríos, Alexandra María. 2015. Migraciones, Género y Salud. Un estudio antropológico del proceso de salud e integración social de las mujeres migrantes extranjeras que 
ejercen la prostitución en Almería, España. Tesis Doctoral. Universidad de Granda, España.

Ruíz, M. 2001. "Igualdad de oportunidades en los servicios sanitarios: sesgos de género como determinante de la estructura de salud de la Comunidad". En Miqueo, Consuelo., Tomás, Concepción; Tejero, Cruz., Barral, María José., Fernández, Teresa y Yago, Teresa (eds.) Perspectivas de género en salud. Fundamentos científicos y socioprofesionales de diferencias sexuales no previstas. Madrid: Minerva. 163-178.

Salcedo, Diana. 2013. "Género, derechos de las víctimas y justicia transicional: Retos en Colombia”. Paz y Conflictos, 6:124-155 Granada.

Távora, Ana. 2001. "El género y los sistemas de referencia en Salud Mental”. En Miqueo, Consuelo., Tomás, Concepción; Tejero, Cruz., Barral, María José., Fernández, Teresa y Yago, Teresa (eds.) Perspectivas de Género en Salud. Fundamentos científicos y socioprofesionales de diferencias sexuales no previstas. Madrid: Minerva. 199-222.

Valls_llobet, Carmen. 2001. "El estado de la investigación en salud y género". En Miqueo, Consuelo., Tomás, Concepción; Tejero, Cruz., Barral, María José., Fernández, Teresa y Yago, Teresa (eds.) Perspectivas de género en salud. Fundamentos científicos y socio profesionales de diferencias sexuales no previstas. Grupo de Salud del Seminario Interdisciplinar de Estudios de la Mujer de la Universidad de Zaragoza. Minerva Ediciones.

2006. Mujeres invisibles. Barcelona: De bolsillo.

2011. Mujeres, Salud y Poder. Madrid: Cátedra.

Weine, S., Golobof, A., Bahromov, M., Kashuba, A., Kalandrarov, T y Jonbekov, J.S. 2013. "Female migrant sex workers in Moscow: gender and power factors and HIV risk". Women Health, 53(1):56-73.

Women's Link Worldwide. 2018. "La audiencia ordena investigar al psicólogo acusado de odio hacia las mujeres". https://www.womenslinkworldwide.org/buscador?s=delito+de+odio $\left[\begin{array}{ll}14 & \text { abril }\end{array}\right.$ 2019].

Wong, M., Chan, R., Tan, H., Yong, E., Lee, L y Cutter, J. 2012. "Sex work and risky sexual behaviors among foreign entertainment workers in urban Singapore: findings from Mystery Client Survey". J Urban Health 9723-5, 89(6):1031-44. 